[7] L. F. Mollenauer, J. P. Gordon, and M. N. Islam, "Soliton propagation in long fibers with periodically compensated loss," IEEE J. Quantum Electron., vol. QE-22, pp. 157-173, 1986.

[8] S. M. J. Kelly, K. Smith, K. J. Blow, and N. J. Doran, "Average soliton dynamics of a high-gain erbium fiber laser," Opt. Lett., vol. 16, pp. 1337-1339, 1991

[9] K. J. Blow, N. J. Doran, and D. Wood, "Generation and stabilization of short soliton pulses in the amplified nonlinear Schrodinger equation," J. Opt. Soc. Amer. B, vol. 5, pp. 381-391, 1988.
[10] J. Satsuma and N. Yajima, "Initial value problems of one-dimensional self-modulation of nonlinear waves in dispersive media," Prog. Theor. Phys. Suppl., no. 55, pp. 284-305, 1974.

[11] M. Ding and K. Kikuchi, "Analysis of soliton transmission in optical fibers with the soliton self-frequency shift being compensated by distributed frequency dependent gain," IEEE J. Photon. Technol. Lett., vol. 4, pp. 497-500, May 1992.

[12] R. H. Stolen and E. P. Ippen, "Raman gain in glass optical waveguides," Appl. Phys. Lett., vol. 22, pp. 276-278, 1973.

\title{
All-Optical Synchronization and Frequency Division of Mode-Locked Pulses
}

\author{
Paul Phelan, Gerald Farrell, and John Hegarty
}

\begin{abstract}
We report all-optical synchronization and frequency division of mode-locked input signals, using a selfpulsating laser diode. The performance of mode-locked and sinusoidal input signals are compared. Synchronization and frequency division of the mode-locked signals is found to be easier to achieve. This result suggests that significant application may be found for this system.
\end{abstract}

$\mathrm{T}$ HERE is now a great deal of interest in functions which can be performed optically, in order that the limitations of electronics can be overcome, and some of the advantages of optics can be exploited. Optical timing extraction is one such function which is currently attracting attention. One approach to optical timing extraction is to use a self-pulsating laser diode to lock onto an input optical data signal [1], [2]. The data signals reported so far have been RZ and NRZ. Recently, we have reported an extension of this system to perform all optical frequency division using a purely sinusoidal clock signal [3].

It has also recently been shown that mode-locked laser diodes are promising candidates for large fan-out optical clock distribution [4]. Even though the large potential bandwidth of such short duration pulses has not as yet been exploited, there is a considerable advantage for these systems, due to the very low jitter associated with mode-locked laser sources. This letter reports all optical synchronization and frequency division for the case of a mode-locked input signal, and compares the performance with that using the purely sinusoidal input.

The experimental setup is very similar to that reported in [3]. The system consists of a tunable source of light

Manuscript received March 12, 1992.

The authors are with Optronics Ireland Research Centre, Department of Physics, Trinity College, Dublin 2, Ireland.

IEEE Log Number 9201889. signals, and a self-pulsating laser diode. The tunable source is a grating external-cavity laser intensity modulated at a controllable frequency. The output of this signal laser is injected into the self-pulsing laser. The self-pulsing laser is a two-section laser whose self-pulsation frequency can be controlled by the combined drive to the two sections [5]. In order to obtain synchronization for a mode-locked input signal, the grating laser is actively mode-locked at the fundamental of the cavity roundtrip frequency in the region of $456 \mathrm{MHz}$. The pulse duration, monitored on a $20 \mathrm{GHz}$ p-i-n photodiode, can be maintained at less than 75 ps by adjustment of the mode-locking conditions, such as bias current, RF frequency, and power level. Single clean pulses are obtained for a dc bias of $11.6 \mathrm{~mA}$ modulated at $456.39 \mathrm{MHz}$ with $+22 \mathrm{dBm}$. With no modulation applied, the grating laser $I_{\text {th }}=34$ $\mathrm{mA}$. The wavelength of this laser is chosen to match a longitudinal mode of the self-pulsing laser [4].

The results of the synchronization for both mode-locked and sinusoidal inputs are shown in Fig. 1. Fig. 1(a) shows the synchronized output of the self-pulsing laser (upper trace) and the input mode-locked signal (lower trace), detected by a $1.5 \mathrm{GHz}$ avalanche photodiode (APD), and displayed on a sampling oscilloscope. This was measured for the case of an average input power to the self-pulsing laser of $4.23 \mu \mathrm{W}$. Very good synchronization is observed, as can be seen from the traces. Fig. 1(b) shows the synchronized output of the self-pulsing laser in the case of a sinusoidal input signal for a power input of $65.0 \mu \mathrm{W}$. Good synchronization is again observed but the quality of the output pulses is not as good as in the cased of the mode-locked input. We attribute this degradation to a decrease in synchronization stability.

Synchronization to a mode-locked input can be achieved at significantly lower powers than for a sinusoidal input. 


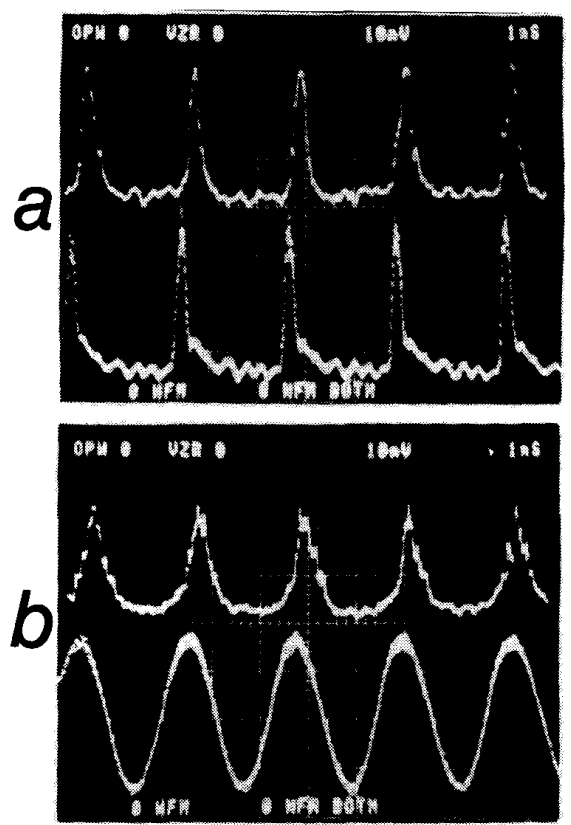

Fig. 1. Synchronization of mode-locked and sinusoidal input signals. Upper trace of (a) is the synchronized output of the self-pulsing laser for the mode-locked input shown in the lower trace. The traces in (b) are for sinusoidal input (upper: self-pulsing laser output, lower: sinusoidal input).

To investigate this we have used a spectrum analyzer to monitor the noise floor power level relative to the power level at the synchronized frequency as a function of the input optical power. In a previous paper we have shown that this is a good measure of the degree of synchronization occurring. The results of this measured power dependence for identical conditions (frequency, wavelength, absorber bias), are presented in Fig. 2. We have plotted the noise floor relative to the peak power level of the locked signal, as a function of the input optical power. In both cases the relative noise floor level decreases as the input optical power is increased. Saturation occurs above a certain input optical power. The input power required for mode-locked signals, however, is smaller by almost an order of magnitude over that for sinusoidal signals. In addition, the saturated noise floor level, which is a measure of the quality of the synchronization is better by almost $10 \mathrm{~dB}$. We can explain this and, hence, the difference in the quality of synchronization between the modelocked and sinusoidal inputs shown in Fig. 1 on the basis of the different harmonic content of the two inputs. Fig. $3(a)-(c)$ shows the intensity noise spectra of the free-running self-pulsating laser with no external optical injection, the mode-locked grating laser, and the sinusoidally modulated grating laser, respectively. The mode-locked laser and the self-pulsing laser possess strong harmonics which overlap each other, while the sinusoidal signal has only the fundamental harmonic. The improved synchronization which is observed for mode-locked signals can be explained as follows. In the case of a sinusoidal input, synchronization can occur only through the overlap of a

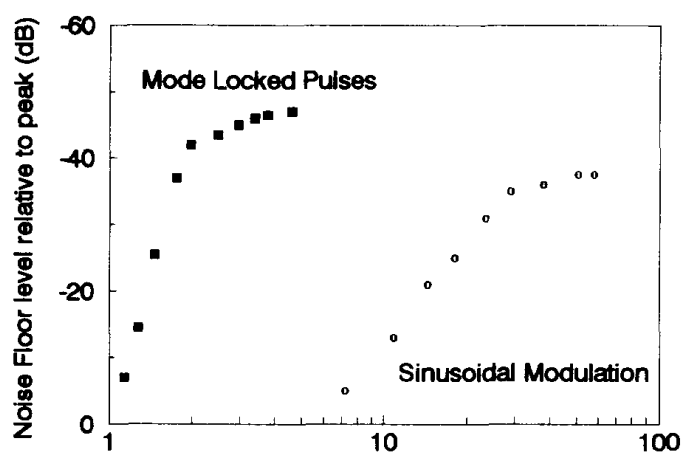

Fig. 2. Noise floor level relative to peak level of the synchronized self-pulsing laser output signal as a function of the injected optical power for mode-locked and sinusoidal input signals.
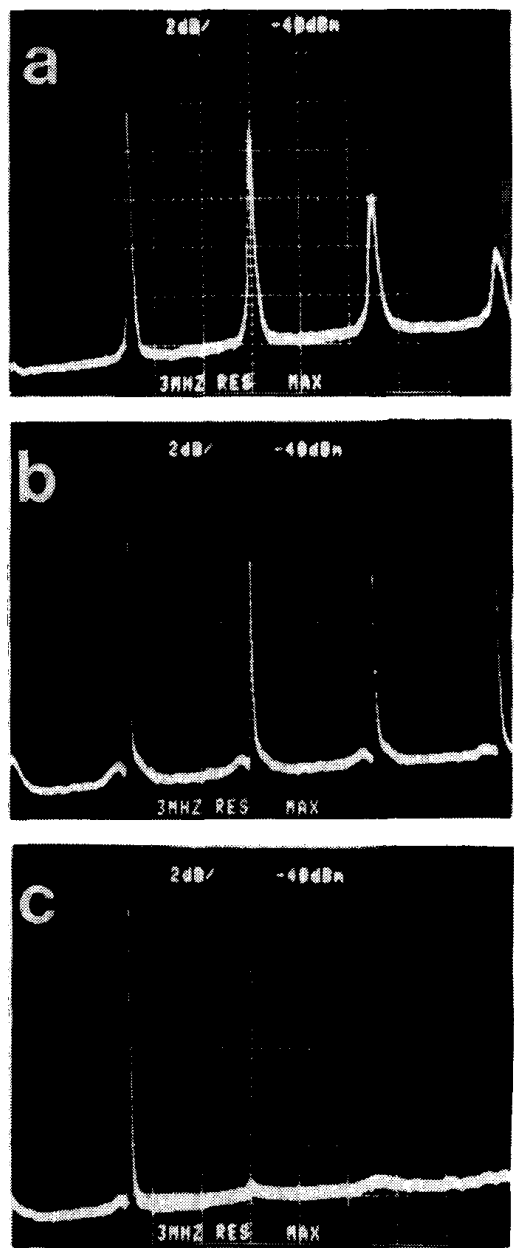

Fig. 3. Intensity noise spectrum from 0 to $1.8 \mathrm{GHz}$ of the detected signal, displayed on a spectrum analyzer. (a) Free running self-pulsing laser (i.e., no injection), (b) mode-locked grating laser, and (c) sinusoidally modulated grating laser.

single harmonic of the self-pulsating laser, in this case the fundamental. In the case of a mode-locked input, not only the fundamental but all of the self-pulsation harmonics 


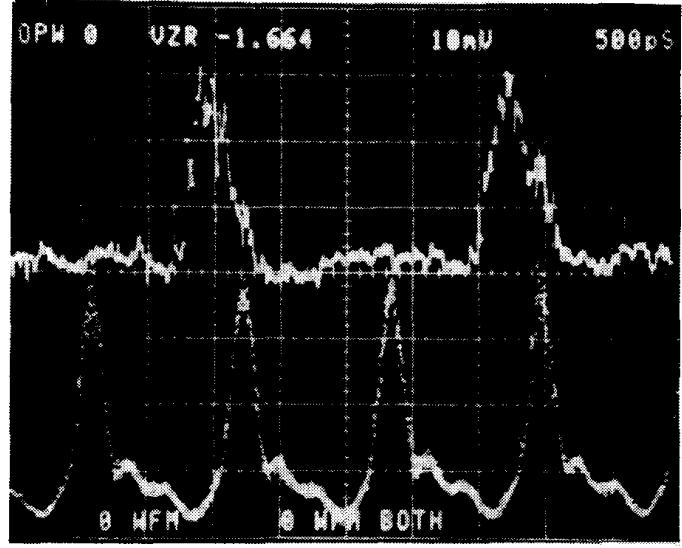

Fig. 4. Frequency division (with synchronization) of mode-locked input signals. Upper trace: synchronized self-pulsing laser output at $510 \mathrm{MHz}$ lower trace: input grating laser mode-locked at $1020 \mathrm{MHz}$.

are overlapped by mode-locked harmonics reducing the power required for synchronization. As in [3], the existence of self-pulsing higher harmonics suggests that an input signal with a fundamental modulation frequency at any of the harmonics will lock the self-pulsing laser and lead to frequency division.

In order to unambiguously demonstrate optical frequency division of a mode-locked input signal, the grating laser cavity length was reduced, and mode-locked at its fundamental frequency of about $1020 \mathrm{MHz}$. Once again the pulses monitored on the $20 \mathrm{GHz}$ detector were maintained at less than $75 \mathrm{ps}$, and coupled into the self-pulsing laser. Fig. 4 illustrates the output from the self-pulsing laser (upper trace), and the input mode-locked source (lower trace). For a similar relative noise floor level, it was again found that there is as an order of magnitude improvement in synchronization over the case of sinusoidal input. This can now be easily understood in terms of the overlapping of the intensity noise spectrum at the higher harmonics. We have also observed an increase in the minimum power required for frequency division with synchronization over that required for synchronization only (1:1). This is due to the fact that the second harmonic of the self-pulsing laser is reduced by $2.5 \mathrm{~dB}$ from the fundamental. In addition only the even harmonics of the self-pulsing laser are overlapped with the mode-locked spectrum.

In conclusion, we have demonstrated all-optical synchronization and frequency division of a mode-locked laser input to a self-pulsing laser diode. The minimum mode-locked power required for synchronization is much less than in the case of a sinusoidal input. We attribute this to the strong higher harmonics of the mode-locked laser which overlap with those of the self-pulsation to produce the synchronization and division. This result shows that all-optical timing extraction and synchronized frequency division of mode-locked pulses is an attractive prospect for large fan-out systems such as clock distribution and demultiplexing of high-speed time division multiplexed signals.

\section{ACKNOWLEDGMENT}

We would like to thank Dr. M. Robertson of BT Laboratories for providing the laser diodes used in this work and A. Stregge of AT \& T Bell Laboratories who provided the APD.

\section{REFERENCES}

[1] M. Jinno and T. Matsumoto, "Optical retiming regenerator using $1.5 \mu \mathrm{m}$ multi-electrode DFB LDs," Electron. Lett., vol. 25, pp. $1332-1333,1988$.

[2] P. Barnsley and H. Wickes, "All optical clock recovery from 2.5 GBit/s NRZ data using a self pulsating $1.56 \mu \mathrm{m}$ laser diode," Electron. Lett., vol. 28, pp. 4-5, 1992.

[3] G. Farrell, P. Phelan, and J. Hegarty, "All-optical synchronization with frequency division using a self-pulsating laser diode," Electron. Lett., vol. 28, pp. 738-739, Feb. 1992.

[4] P. Delfyett, D. Hartman, and S. Zuber Ahmad, "Optical clock distribution using a mode-locked semiconductor laser diode system," J. Lightwave Technol., vol. 9, pp. 1646-1649, 1991.

[5] G. Farrell, P. Phelan, and J. Hegarty, "Self-pulsation operating regime for absorber of twin section laser diode," Electron. Lett., vol. 27, pp.1403-1404, 1991. 